\title{
Minimal important difference and patient acceptable symptom state for pain, Constant-Murley score and Simple Shoulder Test in patients with subacromial pain syndrome
}

Kari Kanto ${ }^{1 \dagger}$, Tuomas Lähdeoja ${ }^{2 \dagger}$, Mika Paavola $^{2}$, Pasi Aronen ${ }^{3}$, Teppo L. N. Järvinen², Jarkko Jokihaara ${ }^{4}$, Clare L. Ardern ${ }^{5}$, Teemu V. Karjalainen ${ }^{6}$ and Simo Taimela ${ }^{2^{*}}$

\begin{abstract}
Background: The results of clinical trials should be assessed for both statistical significance and importance of observed effects to patients. Minimal important difference (MID) is a threshold denoting a difference that is important to patients. Patient acceptable symptom state (PASS) is a threshold above which patients feel well.

Objective: To determine MID and PASS for common outcome instruments in patients with subacromial pain syndrome (SAPS).
\end{abstract}

Methods: We used data from the FIMPACT trial, a randomised controlled trial of treatment for SAPS that included 193 patients. The outcomes were shoulder pain at rest and on arm activity, both measured with the 0-100 mm visual analogue scale (VAS), the Constant-Murley score (CS), and the Simple Shoulder Test (SST). The transition question was a five-point global rating of change. We used three anchor-based methods to determine the MID for improvement: the receiver operating characteristic (ROC) curve, the mean difference of change and the mean change methods. For the PASS, we used the ROC and 75th percentile methods and calculated estimates using two different anchor question thresholds.

Results: Different MID methods yielded different estimates. The ROC method yielded the smallest estimates for MID: $20 \mathrm{~mm}$ for shoulder pain on arm activity, 10 points for CS and 1.5 points for SST, with good to excellent discrimination (areas under curve (AUCs) from 0.86 to 0.94 ). We could not establish a reliable MID for pain at rest. The PASS estimates were consistent between methods. The ROC method PASS thresholds using a conservative anchor question threshold were $2 \mathrm{~mm}$ for pain at rest, $9 \mathrm{~mm}$ for pain on activity, 80 points for CS and 11 points for SST, with AUCs from 0.74 to 0.83 .

(Continued on next page)

\footnotetext{
* Correspondence: simo.taimela@helsinki.fi

${ }^{\dagger}$ Kari Kanto and Tuomas Lähdeoja contributed equally to this work.

${ }^{2}$ Finnish Centre for Evidence-Based Orthopaedics (FICEBO), Department of

Orthopaedics and Traumatology, University of Helsinki and Helsinki University

Hospital, Topeliuksenkatu 5, HUS, 00029 Helsinki, Finland

Full list of author information is available at the end of the article
}

(c) The Author(s). 2021 Open Access This article is licensed under a Creative Commons Attribution 4.0 International License, which permits use, sharing, adaptation, distribution and reproduction in any medium or format, as long as you give appropriate credit to the original author(s) and the source, provide a link to the Creative Commons licence, and indicate if changes were made. The images or other third party material in this article are included in the article's Creative Commons licence, unless indicated otherwise in a credit line to the material. If material is not included in the article's Creative Commons licence and your intended use is not permitted by statutory regulation or exceeds the permitted use, you will need to obtain permission directly from the copyright holder. To view a copy of this licence, visit http://creativecommons.org/licenses/by/4.0/ The Creative Commons Public Domain Dedication waiver (http://creativecommons.org/publicdomain/zero/1.0/) applies to the data made available in this article, unless otherwise stated in a credit line to the data. 
(Continued from previous page)

Conclusion: We recommend the smallest estimate from different methods as the MID, because it is very unlikely that changes smaller than the smallest MID estimate are important to patients: $20 \mathrm{~mm}$ for pain VAS on arm activity, 10 points for CS and 1.5 points for SST. We recommend PASS estimates of $9 \mathrm{~mm}$ for pain on arm activity, 80 points for CS, and 11 points for SST.

Trial registration: ClinicalTrials.gov NCT00428870 (first registered January 29, 2007).

Keywords: Clinimetrics, minimal important change, MID, MCID, patient accepted symptom state, PASS, responsiveness, Outcome measures, Visual analogue scale (VAS), Simple shoulder test, Constant-Murley score, Pain, Subacromial pain

\section{Background}

The efficacy of interventions is often measured as the mean difference between intervention and control groups, or the differences in proportions of patients who achieve a desired state. It is important to judge whether a difference is important to patients, instead of relying on statistical significance testing to draw conclusions about the importance of research results. To assess whether a desired state has been achieved for an individual patient, continuous outcomes must be dichotomised to "success" and "non-success". In most orthopaedic conditions, the outcomes most important to patients are assessed with patient-reported outcome measures (PROMs), which measure pain, function or (disease-related) quality of life. Important questions remain about their interpretation. In particular, how a certain change in PROM score is perceived by the patients, or at what level of a PROM patients consider themselves well. Concepts like MID and PASS have been developed to better understand how PROM scores reflect patients' perceptions of their pain or disability.

The minimal important difference, MID [1], reflects the threshold at which a difference in a continuous outcome is important to patients, either between groups receiving different treatments or within-group at different time points. It is the smallest difference in the outcome of interest that informed patients or informed proxies perceive important enough to convince the patient and/ or clinician to choose one treatment over another [2]. MID is commonly used to help interpret the clinical importance of the results of a trial or a meta-analysis and inform calculations of numbers needed to treat (NNTs) and sample size estimation. The MID is commonly calculated using intra-individual change in outcomes over time by anchor-based methods. In the anchor-based approach, the MID is established by relating a difference in PROM scores to a small, but important improvement or deterioration captured by an independent measure (external anchor) that is itself, interpretable. MID estimates for a given outcome can vary depending on assessment methods and patient populations [3-6].
Another measure that can help to interpret study results is the patient acceptable symptom state (PASS). The PASS is the highest symptom level at which patients consider themselves well [7]. Improving by at least MID measures "feeling better," whereas reaching the level of at least PASS reflects "feeling good." A patient reaching PASS would typically indicate therapeutic success at the individual level. PASS provides a tool for standardising responder rates in clinical trials. The MID and PASS concepts are complementary. For example, with values MID of $20 \mathrm{~mm}$ and PASS of $20 \mathrm{~mm}$, if an intervention leads to a decrease of pain from VAS $80 \mathrm{~mm}$ to VAS 50 $\mathrm{mm}$, the change is important to the patient (concept of MID) but the patient did not reach a satisfactory state (concept of PASS). Results of a trial could be expressed both as a proportion of improved patients and of patients in a satisfactory state. The definition of the PASS is anchored to the personal experience of the patient feeling well or not, and the PASS threshold for each outcome instrument of interest can be calculated using this answer as an external anchor.

Subacromial pain syndrome (SAPS) is the most common shoulder condition [8-10]. Despite the high prevalence of SAPS, only limited and almost exclusively low credibility data exist on MID and PASS thresholds of outcome instruments in patients with this condition [11]. To our knowledge, two PASS estimates for generic pain have been published for patients with SAPS [12, 13]. In other shoulder conditions, namely patients undergoing shoulder arthroplasty $[13,14]$ and patients with rheumatoid arthritis who are awaiting surgery [15], PASS estimates have been published for the American shoulder and elbow surgeons (ASES) score, the Simple Shoulder Test (SST), Shoulder Pain and Disability Index (SPADI), and the Visual Analog Scale (VAS) pain score.

We used the 2-year follow-up data from the FIMP ACT trial [16] to estimate MID and PASS thresholds for four common shoulder outcome instruments used with patients with SAPS. We employed multiple established methods and data from a relatively large, well established, and uniform patient sample. The outcomes included three patient reported outcome measures - 
shoulder pain at rest, shoulder pain on arm activity (both measured using the visual analogue scale) and the Simple Shoulder Test [17]. The Constant-Murley score [18] consists of patient-reported and outcome assessormeasured components.

\section{Materials and methods}

\section{Data source and study population}

FIMPACT is a randomised, placebo-surgery controlled three-arm efficacy trial of subacromial decompression for treating SAPS. The trial was conducted at three orthopaedic clinics in Finland. One hundred ninetythree patients aged 35 to 65 years with SAPS were randomised to arthroscopic subacromial decompression (ASD), diagnostic arthroscopy (DA) or exercise therapy (ET), and followed for 24 months. At the eligibility screening visit, an experienced shoulder surgeon examined the patients to rule out shoulder instability, rotator cuff rupture, frozen shoulder or other causes of shoulder symptoms. All potentially eligible participants had standard $\mathrm{x}$-rays and MRI to rule out rotator cuff rupture and other shoulder pathology. Baseline characteristics of participants are presented in Table S1 in the supplementary appendix and full details of the study can be found in the original articles $[16,19]$.

\section{Data time points}

Pain and global rating of change (GRC) were collected at baseline, 6-, 12- and 24-month follow-ups; SST and Constant-Murley score were measured at baseline, 6and 24-month follow-ups.

\section{Outcome instruments of interest}

\section{Pain at rest and pain on arm activity}

Shoulder pain intensity during the previous $24 \mathrm{~h}$ was assessed on a $100 \mathrm{~mm}$ visual analogue scale (VAS) ranging from 0 (no pain) to 100 (extreme pain) (Fig. S1 in the supplementary appendix). Pain at rest and on arm activity were measured separately.

\section{Shoulder function instruments Constant-Murley score and Simple Shoulder Test}

The Constant-Murley score [18] comprises measures of capacity (range of motion and strength) and subjective parameters (pain assessment, work load, and leisure time activities), which yield a score ranging from 0 (worst) to 100 (best). Although the Constant-Murley score is one of the most frequently cited instruments, it does not have convincing evidence for its psychometric properties [20].

The Simple Shoulder Test (SST) [17], consists of 12 questions of shoulder status and function, with yes (1) or no (0) response options. Answers are summed for a score ranging from 0 to 12 , with maximum score indicating normal shoulder function. The Simple Shoulder
Test has good evidence in support of internal consistency, reliability, structural validity, hypothesis testing, and responsiveness [20].

\section{Global rating of change}

Participants were asked their subjective satisfaction to treatment outcome relative to baseline at the 6-, 12- and 24-month follow-up visits on a five-point global rating of change (GRC) scale (Table 1).

\section{Data analysis for MID}

We used the GRC as the anchor question for calculating the MID. An adequate transition anchor should correlate to the change in outcome, and ideally correlate equally, but in opposite directions to the scores of outcomes at baseline and at follow-up time points (post scores) [21]. The correlation to change should be larger than the correlation to post scores when the GRC captures true change [22]. To explore this, we calculated the correlation coefficients (Spearman's rho) for the GRC answers at different time points and baseline scores, the GRC and each of the respective post scores and the post scores of the combined dataset, and the GRC and the change scores of the outcomes, also at follow-up time points and the combined dataset. 95\% CIs were calculated by bootstrapping 1000 samples for the correlations between the anchor and relevant scores.

We used three approaches to determine the MID for improvement: 1) the ROC method, 2) the mean difference of change (MDoC) method and 3) the mean change (MC) method.

For the ROC method [23], we dichotomised the GRC to improved (responses 1-3; Table 1) and no change (response 4; Table 1). Participants with response worse (response 5; Table 1) were excluded from the ROC analyses to obtain MID estimates for improvement [24]. Because very few patients deteriorated, we could not estimate MIDs for worsening. We used the closest point to top left corner method to choose the cut-off value for the outcome, maximising specificity and sensitivity [25]. For the target measures, we calculated change from baseline to each follow-up point.

Table 1 Global Rating of Change response options ${ }^{a}$

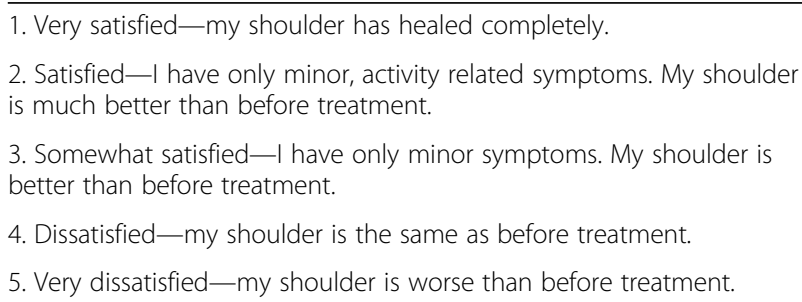

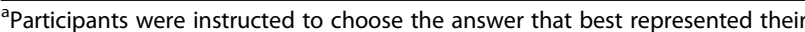
current situation. The answer options 1-5 have been translated from Finnish to English 
To evaluate how well each measure could discriminate between those who were improved and those who were not improved, we calculated the area under the ROC curve (AUC). We determined the confidence intervals for AUC using DeLong's method [26]. The area ranges from 0.5 (no accuracy in distinguishing improved from not improved) to 1.0 (perfect accuracy) $[27,28]$. In musculoskeletal conditions, AUC values between 0.7 and 0.8 are acceptable, and value greater than 0.8 is considered to have good to excellent discrimination [29].

In the $\mathrm{MDoC}$ method, we calculated the mean difference of the change scores of each outcome from baseline to the follow-up time point (with 95\% CIs) between the participants who answered" Somewhat satisfied" and" Dissatisfied" (responses 3 and 4; Table 1). In the MC method, we determined the mean of the change scores from baseline to the follow-up time points (with 95\% CIs) of those who reported" Somewhat satisfied" (response 3; Table 1). With the MDoC and MC methods, the $95 \%$ CIs were calculated by bootstrapping 1000 samples for the MID values.

We combined the data across all time points $(6,12$, 24 months) and used the whole dataset irrespective of treatment for analyses to provide an estimate derived from a larger number of GRC-outcome pairs. We explored the ROC curves, and MID and PASS estimates at different time points and found them to be very similar, supporting our decision to pool data for our primary analysis. To explore whether the different treatments affected the MIDs, we performed sensitivity analyses and calculated MIDs for patients who underwent surgery (ASD and DA groups combined) and for patients who received exercise therapy. In the FIMPACT trial, the blinding between ASD and DA held well, and the patients in both ASD and DA groups subjectively underwent "surgical treatment".

\section{Data analysis for PASS}

For PASS, we used the ROC and the 75th percentile [30] methods for the combined dataset. The ROC method was used similarly as in MID. We used the closest point to top left corner method [25] to determine the cut-off point and the AUCs were used to evaluate how well each measure could discriminate between participants who reported "Very satisfied, my shoulder has healed completely" and the rest of the cohort (responses 2-5, Table 1). In 75th percentile method, PASS was defined as the 25th percentile score for Constant-Murley score and Simple Shoulder Test, and 75th percentile score for pain VASs from the distribution of the patients who answered: "Very satisfied, my shoulder has healed completely". Because the choice whether to use GRC 1 only or both 1 and 2 is debatable, we also calculated the PASS thresholds between participants who reported
"Very satisfied, my shoulder has healed completely" and "Satisfied-I have only minor, activity related symptoms. My shoulder is much better than before treatment." (responses 1-2, Table 1) and the rest of the cohort (responses 3-5, Table 1).

\section{Compliance with research ethics standards}

This study was conducted in compliance with good clinical practice, and the Declaration of Helsinki.

\section{Results}

We analysed data from 193 patients. Due to missing data items, the number of available GRC-outcome pairs varied at each time point (Table 2).

To test the adequacy of our transition anchor, we calculated the correlations to the baseline values and outcomes at each time point. The correlations between the GRC and baseline values were close to zero (Table S2 in the supplementary appendix). The correlations between the GRC and post scores in the combined data were for pain at rest $-0.57(-0.63$ to -0.50$)$, for pain on activity -0.63 ( -0.69 to -0.56$)$, for Constant-Murley score 0.63 (0.55 to 0.69$)$ and for Simple Shoulder Test $0.62(-0.55$ to 0.69 ). The correlations between the GRC and change scores in the combined data were for pain at rest -0.25 $(-0.32$ to -0.16$)$, for pain on activity $-0.53(-0.59$ to 0.47), for Constant-Murley score 0.57 (0.49 to 0.64) and for Simple Shoulder test 0.49 (0.40 to 0.57$)$. The correlations to post scores and change scores at individual time points are presented in Table S2 in the supplementary appendix. The correlation between GRC and change of pain at rest was very low, whereas the correlations between the GRC and change of other outcomes were adequate. The correlations to post scores were larger than the correlations to change.

\section{MID estimates}

MIDs based on receiver operating characteristic method

MID estimates from the ROC analysis with their characteristics are presented in Table 3. In the ROC analysis, MID for Constant-Murley score had excellent discrimination (AUC), while MIDs for pain on arm activity and SST showed good discrimination. Discrimination improved with the follow-up time (Table S3 in the supplementary appendix). MID for pain at rest did not discriminate well. The ROC curves are presented in Fig. S2 in the supplementary appendix.

\section{MIDs based on mean difference of change and mean change methods}

MIDs with 95\% CIs based on the MDoC and MC methods are presented in Table 4. MID values with 95\% CIs from data at each timepoint can be found in the Table S4 in the supplementary appendix. 
Table 2 Numbers of data pairs

\begin{tabular}{lllll}
\hline & $\mathbf{6}$ months & $\mathbf{1 2}$ months & 24 months & Combined \\
\hline Pain at rest & 179 & 176 & 184 & 539 \\
Pain on arm activity & 179 & 175 & 184 & 538 \\
Constant-Murley score & 179 & - & 179 & 358 \\
Simple Shoulder Test & 179 & - & 180 & 359 \\
\hline
\end{tabular}

- denotes that outcomes were not measured at the time point

MID values calculated by the MDoC and $\mathrm{MC}$ methods were larger than the MID values from the ROC analysis, especially for the Constant-Murley score by a factor of two: In the primary analysis 9.5 points with ROC method; 23 points with MDoC method; 24 points with MC method. Similar results were obtained in the analyses of separate time points. The estimates of MID values calculated from data of patients who underwent surgery were similar to MIDs derived from patients who received exercise therapy, but the confidence intervals were wide and the ROC curves were not ideal, making these subgroup results unreliable.

\section{PASS estimates}

Estimates for PASS derived by the ROC and the 75th percentile methods are presented in Table 5. The AUCs had acceptable to good discrimination. The ROC curves are presented in Fig. S3 in the supplementary appendix. The PASS estimates provided by the 75 th percentile method were almost identical to the values from the ROC analysis.

\section{Discussion}

Our MID estimates for pain on arm activity, the Constant-Murley score, and the Simple Shoulder Test appear trustworthy: In the ROC analysis they showed good (pain on arm activity and Simple Shoulder Test) or excellent (Constant-Murley score) discrimination between patients who considered themselves improved or not improved. The correlations to change scores were adequate for these three outcomes, but the correlations to post scores were slightly larger than the correlations to change scores. MID values derived for pain at rest do not appear useful in this patient population. When interpreting trial results, the smallest credible estimate from different methods for MID sets the low limit for the MID, as changes smaller than the smallest MID estimate are very unlikely to be important to patients. The likely best MID estimates were $20 \mathrm{~mm}$ for pain VAS on arm activity, 10 points for Constant-Murley score and 1.5 points for Simple Shoulder Test.

The PASS estimates using GRC 1 answer only for pain on arm activity $(9 \mathrm{~mm})$, Constant-Murley score (81 points), and Simple Shoulder Test (11 points) were consistent between methods and showed good discrimination. Using GRC answers $1+2$, the PASS estimates were also consistent between methods and the AUCs were better or similar to the analysis using answer option 1 only. The PASS estimates with GRC $1+2$ were 8 for pain at rest, 26 for pain on arm activity, 69 for the Constant-Murley score and 9 for the Simple Shoulder Test. We recommend using more conservative of the estimates for PASS values, which we think certainly represents a state of being well.

In line with previous findings [3, 4], our analysis based on the FIMPACT trial data found high variability of the MID estimates both between methods and outcome instruments. These findings showcase the challenges of the MID concept and highlight the need for deep understanding of the instruments, statistical methods, and differences in patient populations when applying the MID results in clinical practice.

\section{Strengths and weaknesses of the study}

We used multiple established methods to estimate the MID and PASS values in a relatively large patient sample with high adherence to follow-up ( $92 \%$ at 24 months). Our study population was exceptionally well established and uniform: a robust clinical examination by highly experienced orthopaedic surgeons ensured that participants had clinical findings consistent with SAPS, and magnetic resonance imaging with intra-articular contrast agent (MRA) was used to exclude other shoulder

Table 3 MID estimates from the ROC analysis

\begin{tabular}{lllll}
\hline Outcome measure & MID & Sensitivity & Specificity & AUC (95\% Cl) \\
\hline Pain at rest (VAS 0-100) & 13 & 0.63 & 0.72 & $0.72(0.64$ to 0.81$)$ \\
Pain on arm activity (VAS 0-100) & 20 & 0.90 & 0.78 & $0.86(0.82$ to 0.91$)$ \\
Constant-Murley score (0-100) & 10 & 0.90 & 0.89 & $0.94(0.92$ to 0.97$)$ \\
Simple Shoulder Test (0-12) & 1.5 & 0.83 & 0.78 & $0.87(0.82$ to 0.92$)$ \\
\hline
\end{tabular}


Table 4 MID estimates from the mean difference of change (MDoC) and mean change (MC) analyses

\begin{tabular}{lll}
\hline Outcome measure & Method & MID (95\% Cl) \\
\hline Pain at rest (VAS 0-100) & MDoC & 16 (7 to 27$)$ \\
Pain on arm activity (VAS 0-100) & MC & 23 (18 to 27$)$ \\
& MC & 24 (14 to 34) \\
Constant-Murley score (0-100) & MDoC & 24 (20 to 32$)$ \\
Simple Shoulder Test (0-12) & MC & 23 (19 to 27$)$ \\
& MDoC & $2.3(1.4$ to 3.3$)$ \\
& MC & $2.7(2.1$ to 3.3$)$ \\
\hline
\end{tabular}

pathology. The strict inclusion criteria may limit the generalisability of our results to other shoulder conditions.

The anchor question we used for determining the PASS was not verbatim the recommended PASS question [31]. The FIMPACT trial was initiated prior to the publication of the PASS concept and the recommended anchor question, forcing us to use the best available PASS anchor. The choice between GRC 1 and $1+2$ to represent a satisfied patient is not clear, so we calculated the PASS values for both choices. We recommend the PASS estimates calculated with GRC answer 1 only, thinking that erring on the side of caution would be the wise choice here. While acknowledging that our choice can provide a conservative threshold for PASS, we are confident that patients are satisfied with a "completely healed shoulder" and that this response option truly represents a state of being well.
Given the relatively long interval between the baseline and first follow-up, risk of recall bias is obvious. This notion is supported by very low correlations between the transition item and baseline and lower correlations to change scores than to the post scores [21, 22]. This is an inherent weakness of the GRC in a setting where the condition needs longer to evolve than a reliable recall time frame [22]. The AUCs generated by the ROC method had good to excellent discrimination. Also, each of the GRC response options contained a description of satisfaction to the change (and some also a statement of state) and this could affect patient responses compared to a pure satisfaction or change questions. However, the patients were very symptomatic at the baseline, and we think that the answer options capture change in their wording, and the best category also represents a satisfactory state.

\section{Comparison to previous studies}

The method of determining MID affected the values in our study $[3,4]$. The lowest cut-offs were obtained with the ROC method ( $20 \mathrm{~mm}$ for pain on arm activity, 10 points for Constant-Murley score and 1.5 points for Simple Shoulder Test) and the highest with the MC method (26, 23, and 2.7, respectively).

A recent systematic review of anchor-based MIDs for improvement in patient-reported outcomes provided MID estimates for mixed shoulder conditions [11]. There was large variation in reported values between studies. The median estimate for MID concerning pain at rest measured with VAS was $30 \mathrm{~mm}$ and for pain VAS on arm activity was $21 \mathrm{~mm}$ [11]. Our MID estimate

Table 5 PASS estimates

\begin{tabular}{|c|c|c|c|c|c|}
\hline \multirow[t]{2}{*}{ Outcome measure } & \multirow{2}{*}{$\begin{array}{l}\text { 75th percentile method } \\
\text { PASS }\end{array}$} & \multicolumn{4}{|c|}{ ROC method } \\
\hline & & PASS & Sens & Spec & AUC $(95 \% \mathrm{Cl})$ \\
\hline \multicolumn{6}{|c|}{ Pain at rest (VAS 0-100) } \\
\hline GRC answer 1 & 2 & 2 & 0.71 & 0.69 & 0.74 (0.70 to 0.79$)$ \\
\hline GRC answers $1+2$ & 6 & 8 & 0.79 & 0.75 & $0.83(0.79$ to 0.86$)$ \\
\hline \multicolumn{6}{|c|}{ Pain on arm activity (VAS $0-100$ ) } \\
\hline GRC answer 1 & 10 & 9 & 0.80 & 0.75 & 0.83 (0.79 to 0.88$)$ \\
\hline GRC answers $1+2$ & 29 & 26 & 0.73 & 0.77 & 0.82 (0.78 to 0.86$)$ \\
\hline \multicolumn{6}{|c|}{ Constant-Murley score $(0-100)$} \\
\hline GRC answer 1 & 80 & 81 & 0.73 & 0.74 & 0.78 (0.73 to 0.83$)$ \\
\hline GRC answers $1+2$ & 71 & 69 & 0.81 & 0.75 & 0.85 (0.80 to 0.89$)$ \\
\hline \multicolumn{6}{|c|}{ Simple Shoulder Test (0-12) } \\
\hline GRC answer 1 & 11 & 11 & 0.68 & 0.81 & $0.80(0.75$ to 0.85$)$ \\
\hline GRC answers $1+2$ & 9 & 9 & 0.81 & 0.70 & 0.83 (0.78 to 0.87 ) \\
\hline
\end{tabular}

GRC answer 1: Very satisfied-my shoulder has healed completely

GRC answer 2: Satisfied-I have only minor, activity related symptoms. My shoulder is much better than before treatment 
for pain VAS on arm activity is in line with the systematic review [11], but there is a marked difference in MID estimates for pain at rest. In our study, change scores of pain VAS at rest did not show adequate correlation with the anchor question and the AUCs in the ROC analysis were low, which in our opinion aligns well with the clinical reality that pain at rest is rarely the predominant symptom driving patients with SAPS to seek medical attention. The MID estimate for Constant-Murley score was 8.3 points in the systematic review, which is very similar to our estimate (10 points). The median estimate for SST was 1.8 points [11], again consistent with our result of 1.5 points. Another recent systematic review [32] identified two studies that had assessed MID estimates for Constant-Murley score in patients with rotator cuff tears. The MID estimates were 8 to 10 points.

We identified two studies that attempted to determine PASS estimates for VAS or numeric pain rating scale (NPRS) of patients treated for subacromial pain. Tubach et al. [12] reported PASS estimates for VAS ranging from 16 to $24 \mathrm{~mm}$ in patients treated nonoperatively for "acute rotator cuff syndrome." Tashjian et al. [13] reported a PASS estimate of $30 \mathrm{~mm}$ for shoulder pain VAS for patients with rotator cuff disease treated without surgery. Neither study reported separate values for pain at rest or pain on arm activity. Nevertheless, our recommended estimates are markedly lower, probably due to use of only the "very satisfied, completely healed" category as the anchor for PASS.

We were not able to identify studies reporting PASS estimates for Constant-Murley score or Simple Shoulder Test in patients with subacromial pain syndrome.

\section{Meaning of the study}

The smallest trustworthy estimate from different methods for MID can be used because anything less than the smallest MID estimate should be interpreted as unimportant to the patient. There may be settings where using the highest estimate is useful, for example, in a superiority trial where there is a large difference between treatments the higher limit for MID might be a good choice as a threshold for "unequivocal effectiveness.". In our study, the ROC method provided the smallest estimates and discriminated well between those who considered themselves improved from those not improved. Pain at rest showed poor correlation with the anchor question and change score and low ability to discriminate, reflecting its low usefulness in this patient population.

Our PASS estimates for pain at rest and pain on arm activity, Constant-Murley score, and Simple Shoulder Test were consistent across methods and showed good to excellent discrimination between those who considered themselves well from those who did not. We chose to recommend using the estimates derived from analysis using GRC answer 1 only, but it is likely to be conservative, as the acceptable symptoms state may include some minor symptoms also. When the PASS estimates are used to interpret study results, depending on the study setting and characteristics of the patient population, the estimates derived using GRC $1+2$ can be applicable as well.

\section{Unanswered questions and future research}

The MID estimates vary widely, depending on assessment methods and patient populations [33]. Change appears to be baseline dependent: people with more severe symptoms need to experience a greater change to consider their condition improved $[30,34]$ and the results are sensitive to the time point and anchor questions used. Future research topics include determining and then standardising the best method(s) - including the anchor question - for estimating the MID. Qualitative approaches might also have a place in future research [35].

\section{Conclusion}

Different methods provided different estimates for MIDs. We recommend MID estimates for patients with subacromial pain as follows: $20 \mathrm{~mm}$ for pain VAS on arm activity, 10 points for Constant-Murley score and 1.5 points for Simple Shoulder Test. We could not establish a reliable MID for changes in pain at rest in this patient population. We recommend PASS estimates of 9 $\mathrm{mm}$ for pain on arm activity, 80 points for ConstantMurley score, and 11 points for Simple Shoulder Test.

\begin{abstract}
Abbreviations
AUC: Area under curve; CS: Constant-Murley score; GRC: Global rating of change; MC: Mean change; MDoC: Mean difference of change; MID: Minimal important difference; NNT: Numbers needed to treat; NPRS: Numeric pain rating scale; PASS: Patient acceptable symptom state; ROC: Receiver operating characteristic; SAPS: Subacromial pain syndrome; SPADI: Shoulder Pain and Disability Index; SST: Simple Shoulder Test; VAS: Visual analogue scale
\end{abstract}

\section{Supplementary Information}

The online version contains supplementary material available at https://doi. org/10.1186/s12874-021-01241-w.

Additional file 1: Table S1. Baseline characteristics of the participants according to study group. Table S2 Correlations between GRC and baseline, post scores and change scores*. Table S3 MID estimates from the ROC analysis at 6, 12, 24 months. Table S4 MID values calculated by the mean difference of change (MDoC) and the mean change (MC) methods and their respective 95\% confidence intervals (CI). Fig. S1 VAS figure from the original questionnaire (translated from Finnish to English). Fig. S2 MID ROC curves. Fig. S3 PASS ROC curves.

\section{Acknowledgements}

We thank the patients for their participation. We would like to thank the entire FIMPACT investigators study group for the execution of the trial.

\section{Authors' contributors}

KK, TJ, and ST conceived and designed the study, PA, TK, TL and ST executed data analysis, and KK, TL, ST, TK, MP, JJ and CA participated in the analysis 
and interpretation of the data, drafted and revised the article. All authors contributed to final data interpretation and contributed to and approved the final draft of the manuscript. The lead author affirms that this manuscript is an honest, accurate, and transparent account of the study being reported; that no important aspects of the study have been omitted; and that any discrepancies from the study as planned (and, if relevant, registered) have been explained.

\section{Funding}

The FIMPACT trial was supported by the Sigrid Juselius Foundation and the state funding for university level health research (Tampere and Helsink University Hospitals). The funders of the study had no role in study design, data collection, data analysis, data interpretation, or writing of the report. Sponsors had no access to the data and did not perform any of the study analysis. The corresponding author had full access to all the data in the study and had final responsibility for the decision to submit for publication.

\section{Availability of data and materials}

FIMPACT data are not publicly available owing to data privacy issues, but access to the anonymised dataset can be obtained from the corresponding author on reasonable request.

\section{Declarations}

\section{Ethics approval and consent to participate}

The study protocol was approved by the Institutional Review Board of the Pirkanmaa Hospital District (R04200; December 28, 2004) and informed consent was obtained from all participants prior to inclusion in the study. The trial was conducted in accordance with the 1964 Helsinki declaration and its later amendments.

\section{Consent for publication}

Not Applicable.

\section{Competing interests}

All authors have completed the ICMJE uniform disclosure form at www. icmje.org/coi_disclosure.pdf (available on request from the corresponding author) and declare: no support from any organisation for the submitted work other than those described above; All authors declare: no support from any organisation for the submitted work; no financial relationships with any organisations that might have an interest in the submitted work in the previous three years; no other relationships or activities that could appear to have influenced the submitted work.

\section{Author details}

'Finnish Centre for Evidence-Based Orthopaedics (FICEBO), Department of Orthopaedics and Traumatology, Tampere University Hospital, TAYS Hatanpää, Hatanpäänkatu 24, 33900 Tampere, Finland. ${ }^{2}$ Finnish Centre for Evidence-Based Orthopaedics (FICEBO), Department of Orthopaedics and Traumatology, University of Helsinki and Helsinki University Hospital, Topeliuksenkatu 5, HUS, 00029 Helsinki, Finland. ${ }^{3}$ Biostatistics Unit, Faculty of Medicine at University of Helsinki and Helsinki University Hospital, Tukholmankatu 8B, 00290 Helsinki, Finland. ${ }^{4}$ Finnish Centre for Evidence-Based Orthopaedics (FICEBO), Department of Hand and Microsurgery, Tampere University Hospital, Elämänaukio 2, 33520 Tampere, Finland. ${ }^{5}$ Finnish Centre for Evidence-Based Orthopaedics (FICEBO), Division of Physiotherapy, Karolinska Institute, H1 Fysioterapi, 17177 Stockholm, Sweden. ${ }^{6}$ Finnish Centre for Evidence-Based Orthopaedics (FICEBO), Central Finland Central Hospital, Jyväskylä, Keskussairaalantie 19, 40620 Jyväskylä, Finland.

\section{Received: 5 November 2020 Accepted: 24 February 2021} Published online: 06 March 2021

\section{References}

1. Jaeschke R, Singer J, Guyatt GH. Measurement of health status. Ascertaining the minimal clinically important difference. Control Clin Trials. 1989;10(4): 407-15.

2. Schunemann HJ, Puhan M, Goldstein R, Jaeschke R, Guyatt GH. Measurement properties and interpretability of the chronic respiratory disease questionnaire (CRQ). COPD. 2005;2(1):81-9.
3. Terwee CB, Roorda LD, Dekker J, Bierma-Zeinstra SM, Peat G, Jordan KP, et al. Mind the MIC: large variation among populations and methods. J Clin Epidemiol. 2010;63(5):524-34.

4. Mills KA, Naylor JM, Eyles JP, Roos EM, Hunter DJ. Examining the minimal important difference of patient-reported outcome measures for individuals with knee osteoarthritis: a model using the knee injury and osteoarthritis outcome score. J Rheumatol. 2016;43(2):395-404.

5. Devji T, Carrasco-Labra A, Guyatt G. Mind the methods of determining minimal important differences: three critical issues to consider. Evid Based Ment Health. 2020 Aug 24:ebmental-2020-300164. https://doi.org/10.1136/ ebmental-2020-300164. Epub ahead of print. PMID: 32839275.

6. Devji T, Guyatt GH, Lytvyn L, Brignardello-Petersen R, Foroutan F, Sadeghirad B, et al. Application of minimal important differences in degenerative knee disease outcomes: a systematic review and case study to inform BMJ rapid recommendations. BMJ Open. 2017:7(5):e015587.

7. Kvien TK, Heiberg T, Hagen KB. Minimal clinically important improvement/ difference (MCII/MCID) and patient acceptable symptom state (PASS): what do these concepts mean? Ann Rheum Dis. 2007;66(Suppl 3):iii40-1.

8. Mitchell C, Adebajo A, Hay E, Carr A. Shoulder pain: diagnosis and management in primary care. BMJ. 2005;331(7525):1124-8.

9. Harkness EF, Macfarlane GJ, Nahit ES, Silman AJ, McBeth J. Mechanical and psychosocial factors predict new onset shoulder pain: a prospective cohort study of newly employed workers. Occup Environ Med. 2003;60(11):850-7.

10. van der Windt DA, Thomas E, Pope DP, de Winter AF, Macfarlane GJ, Bouter LM, et al. Occupational risk factors for shoulder pain: a systematic review. Occup Environ Med. 2000;57(7):433-42.

11. Hao Q, Devji T, Zeraatkar D, Wang Y, Qasim A, Siemieniuk RAC, et al. Minimal important differences for improvement in shoulder condition patient-reported outcomes: a systematic review to inform a BMJ rapid recommendation. BMJ Open. 2019;9(2):e028777.

12. Tubach F, Dougados M, Falissard B, Baron G, Logeart I, Ravaud P. Feeling good rather than feeling better matters more to patients. Arthritis Rheum. 2006;55(4):526-30

13. Tashjian RZ, Deloach J, Porucznik CA, Powell AP. Minimal clinically important differences (MCID) and patient acceptable symptomatic state (PASS) for visual analog scales (VAS) measuring pain in patients treated for rotator cuff disease. J Shoulder Elb Surg. 2009;18(6):927-32.

14. Tashijian RZ, Hung M, Keener JD, Bowen RC, McAllister J, Chen W, et al. Determining the minimal clinically important difference for the American shoulder and elbow surgeons score, simple shoulder test, and visual analog scale (VAS) measuring pain after shoulder arthroplasty. J Shoulder Elb Surg. 2017;26(1):144-8.

15. Christie A, Dagfinrud H, Garratt AM, Ringen Osnes H, Hagen KB. Identification of shoulder-specific patient acceptable symptom state in patients with rheumatic diseases undergoing shoulder surgery. J Hand Ther. 2011;24(1):53-60 quiz 1.

16. Paavola M, Malmivaara A, Taimela S, Kanto K, Inkinen J, Kalske J, et al. Subacromial decompression versus diagnostic arthroscopy for shoulder impingement: randomised, placebo surgery controlled clinical trial. BMJ. 2018;362:k2860.

17. Matsen FA 3rd, Ziegler DW, DeBartolo SE. Patient self-assessment of health status and function in glenohumeral degenerative joint disease. J Shoulder Elb Surg. 1995:4(5):345-51.

18. Constant CR, Murley AH. A clinical method of functional assessment of the shoulder. Clin Orthop Relat Res. 1987;214:160-4.

19. Paavola M, Malmivaara A, Taimela S, Kanto K, Jarvinen TL, Investigators F. Finnish subacromial impingement arthroscopy controlled trial (FIMPACT): a protocol for a randomised trial comparing arthroscopic subacromial decompression and diagnostic arthroscopy (placebo control), with an exercise therapy control, in the treatment of shoulder impingement syndrome. BMJ Open. 2017;7(5):e014087.

20. Huang H, Grant JA, Miller BS, Mirza FM, Gagnier JJ. A systematic review of the psychometric properties of patient-reported outcome instruments for use in patients with rotator cuff disease. Am J Sports Med. 2015:43(10):2572-82.

21. Guyatt $\mathrm{GH}$, Norman $\mathrm{GR}$, Juniper EF, Griffith LE. A critical look at transition ratings. J Clin Epidemiol. 2002;55(9):900-8.

22. Devji T, Carrasco-Labra A, Qasim A, Phillips M, Johnston BC, Devasenapathy $\mathrm{N}$, et al. Evaluating the credibility of anchor based estimates of minimal important differences for patient reported outcomes: instrument development and reliability study. Bmj. 2020;369:m1714 
23. Hanley JA. Receiver operating characteristic (ROC) methodology: the state of the art. Crit Rev Diagn Imaging. 1989;29(3):307-35.

24. Turner D, Schunemann HJ, Griffith LE, Beaton DE, Griffiths AM, Critch JN, et al. Using the entire cohort in the receiver operating characteristic analysis maximizes precision of the minimal important difference. J Clin Epidemiol. 2009;62(4):374-9.

25. Froud $R$, Abel G. Using ROC curves to choose minimally important change thresholds when sensitivity and specificity are valued equally: the forgotten lesson of pythagoras. theoretical considerations and an example application of change in health status. PLoS One. 2014;9(12):e114468.

26. DeLong ER, DeLong DM, Clarke-Pearson DL. Comparing the areas under two or more correlated receiver operating characteristic curves: a nonparametric approach. Biometrics. 1988;44(3):837-45.

27. Hanley JA, McNeil BJ. A method of comparing the areas under receiver operating characteristic curves derived from the same cases. Radiology. 1983;148(3):839-43.

28. Liang MH. Evaluating measurement responsiveness. J Rheumatol. 1995;22(6): $1191-2$.

29. Wright AA, Cook CE, Baxter GD, Dockerty JD, Abbott JH. A comparison of 3 methodological approaches to defining major clinically important improvement of 4 performance measures in patients with hip osteoarthritis. J Orthop Sports Phys Ther. 2011;41(5):319-27.

30. Tubach F, Ravaud P, Baron G, Falissard B, Logeart I, Bellamy N, et al. Evaluation of clinically relevant changes in patient reported outcomes in knee and hip osteoarthritis: the minimal clinically important improvement. Ann Rheum Dis. 2005;64(1):29-33.

31. Tubach F, Ravaud P, Beaton D, Boers M, Bombardier C, Felson DT, et al. Minimal clinically important improvement and patient acceptable symptom state for subjective outcome measures in rheumatic disorders. J Rheumatol. 2007;34(5):1188-93.

32. Dabija DI, Jain NB. Minimal clinically important difference of shoulder outcome measures and diagnoses: a systematic review. Am J Phys Med Rehabil. 2019;98(8):671-6.

33. Katz NP, Paillard FC, Ekman E. Determining the clinical importance of treatment benefits for interventions for painful orthopedic conditions. J Orthop Surg Res. 2015;10:24.

34. Farrar JT, Young JP Jr, LaMoreaux L, Werth JL, Poole RM. Clinical importance of changes in chronic pain intensity measured on an 11-point numerical pain rating scale. Pain. 2001;94(2):149-58.

35. Staunton H, Willgoss T, Nelsen L, Burbridge C, Sully K, Rofail D, et al. An overview of using qualitative techniques to explore and define estimates of clinically important change on clinical outcome assessments. J Patient Rep Outcomes. 2019;3(1):16.

\section{Publisher's Note}

Springer Nature remains neutral with regard to jurisdictional claims in published maps and institutional affiliations.

Ready to submit your research? Choose BMC and benefit from:

- fast, convenient online submission

- thorough peer review by experienced researchers in your field

- rapid publication on acceptance

- support for research data, including large and complex data types

- gold Open Access which fosters wider collaboration and increased citations

- maximum visibility for your research: over $100 \mathrm{M}$ website views per year

At $\mathrm{BMC}$, research is always in progress.

Learn more biomedcentral.com/submissions 